August 2001

\title{
Determinant Line Bundles and Topological Invariants of Hyperbolic Geometry - Expository Remarks
}

\author{
Andrei A. Bytsenko円, Marcos C. FAlleiros'], Antonio E. \\ GONÇALVES巨, and ZHANNA G. KUZNETSOVA円,
}

Departamento de Fisica, Universidade Estadual de Londrina, Caixa Postal 6001, Londrina-Parana, Brazil

\begin{abstract}
We give some remarks on twisted determinant line bundles and Chern-Simons topological invariants associated with real hyperbolic manifolds. Index of a twisted Dirac operator is derived. We discuss briefly application of obtained results in topological quantum field theory.

\footnotetext{
${ }^{1}$ email: abyts@uel.br

${ }^{2}$ email: faleiros@npd.uel.br

${ }^{3}$ email: goncalve@uel.br

${ }^{4}$ email: jane@fisica.uel.br
} 


\section{Introduction}

The topological invariants have been explicitly calculated for a number of 3-manifolds and gauge groups 11, 2, 3, 4, 5, 6, 77. In dimension three there are two important topological quantum field theories of cohomological type, namely topological $S U(2)$ gauge theory of flat connection and a version of the Seiberg-Witten theory. The twisted $\mathcal{N}=4$ $S U S Y S U(2)$ pure gauge theory (version of the Donaldson-Witten theory) describes the Casson invariant [8] while Seiberg-Witten theory is a $3 \mathrm{~d}$ twisted version of $\mathcal{N}=4$ SUSY U(1) gauge theory with matter multiplet [9, 10]. The both theories can be derived from $4 \mathrm{~d} \mathcal{N}=2 S U S Y S U(2)$ gauge theory $[11]$ corresponding via twist to DonaldsonWitten theory. It would be interesting and natural to investigate dual description of the $\mathcal{N}=2$ theory in low-energy limit. It could provides formulation of invariants of four-manifolds involving elements of the Chern-Simons invariants.

The Chern-Simons invariant $W_{C S}(X ; k)$ weightted by $\exp (\sqrt{-1} k C S(A))$ has to all orders in $k^{-1}=\hbar / 2 \pi \quad(k \in \mathbf{Z})$ an asymptotic stationary phase approximation of the form [12]:

$$
W_{C S}(X ; k)=\sum_{j} W^{(j)}(X ; k) \exp \sqrt{-1} k\left(C S\left(A^{(j)}\right)+\sum_{n=2}^{\infty} C S_{n}\left(A^{(j)}\right) k^{-n}\right)
$$

where $C S\left(A^{(j)}\right)$ is the Chern-Simons secondary characteristic class related to the flat connections $A^{(j)}$ and $C S_{n}\left(A^{(j)}\right)$ are the n-loop quantum corrections coming from the nloop 1-particle irreducible Feynman diagrams.

The semiclassical approximation for the Chern-Simons partition function (quadratic functional) may be expressed by the asymptotics for $k \rightarrow \infty$ of Witten's invariant. This asymptotics leads to a series of $C^{\infty}$ - invariants associated with triplets $\{X ; F ; \xi\}$ with $X$ a smooth homology 3 - sphere, $F$ a homology class of framings of $X$, and $\xi$ an acyclic conjugacy class of ortogonal representations of the fundamental group $\pi_{1}(X)$ [13. In addition the cohomology $H(X ; A d \xi)$ of $X$ with respect to the local system related to Ad $\xi$ vanishes.

Our aim here are designed to provide readers with a brief introduction to the anomaly and index formulas, associated with Chern-Simons invariants and to indicate how these formulas are derived. We shall limit our discussion, in particular, to the real hyperbolic manifolds.

We begin with some conventions which apply throughout. Let $X$ be a locally symmetric Riemannian manifold with negative sectional curvature. Its universal covering $\widetilde{X} \rightarrow X$ is a Riemannian symmetric space of rank one. The group of orientation preserving isometries $\widetilde{G}$ of $\widetilde{X}$ is a connected semisimple Lie group of real rank one and $\widetilde{X}=\widetilde{G} / \widetilde{K}$, where $\widetilde{K}$ is a maximal compact subgroup of $\widetilde{G}$. The fundamental group of $X$ acts by covering transformations on $\widetilde{X}$ and gives rise to a discrete, co-compact subgroup $\Gamma \subset \widetilde{G}$ such that $X=\Gamma \backslash \widetilde{G} / \widetilde{K}$. If $G$ is a linear connected finite covering of $\widetilde{G}$, the embedding $\Gamma \hookrightarrow \widetilde{G}$ lifts to an embedding $\Gamma \hookrightarrow G$. Let $K \subset G$ be a maximal compact subgroup of $G$, then $\Gamma \backslash G / K$ is a compact manifold. For $G=S O(m, 1)\left(m \in \mathbf{Z}_{+}\right), K=S O(m)$. The corresponding symmetric space of non-compact type is the real hyperbolic space $\mathbf{H}^{m}$ of sectional curvature -1 . It's compact dual space is the unit $m$ - sphere. In Sect. 3 we shall be working 
with real compact hyperbolic manifolds with topology $\Gamma \backslash G / K=\Gamma \backslash \mathbf{H}^{2 n}$ (for details see Refs. [14, 15]).

\section{Determinat lines and eta invariants}

The differential geometry of determinant line bundles has been developed in [16] in a special case and in [17, 18] in general. In papers [19, 20] the results on eta invariants (the exsponentiated eta invariant naturally takes values in the determinant line of the boundary) were used to reprove the holonomy formula for determinant line bundles, known as Witten's global anomaly formula [21].

Results which we have considered in the paper hold for any Dirac operator on a Spin ${ }^{\mathbf{C}}$ manifold coupled to a vector bundle with connection (manifolds are assumed spin and its metric is a product near the boundary). On a closed odd dimensional manifold $X$ the Dirac operator $\mathcal{D}_{X}$ is self-adjoint and has discrete spectrum $\operatorname{Spec}\left(\mathcal{D}_{X}\right)$. The eta invariant $\eta_{X}(s)$ of Atiyah-Patodi-Singer [22] by general estimates is converges for $\Re(s)$ sufficiently large. For Dirac operators the meromorphic continuation is analytic for $\Re(s)>-2$ [18]. At $s=0$ the eta invariant is reguliar and we set

$$
\tau_{X}=\exp \left(\pi \sqrt{-1}\left(\eta_{X}\left(0, \mathcal{D}_{X}\right)+\operatorname{dim} \operatorname{Ker} \mathcal{D}_{X}\right)\right) \in \mathbf{C}
$$

The eta invariant $\eta_{X}\left(0, \mathcal{D}_{X}\right)$ is discontinuous in general but the general theory shows that $\tau_{X}$ varies smoothly in families $\left(\left|\tau_{X}\right|=1\right)$.

We recall some standard material on Dirac bundles (for details see, for example, Ref. [23]). Let $\partial X$ be an even-dimensional orientable Riemannian manifold, and let $\mathbf{E} \rightarrow \partial X$ be a hermitian vector bundle equipped with a compatible connection $\nabla$. Let $C \ell(\partial X)$ denote the complexified Clifford bundle. We suppose that there is a bundle map from $C \ell(\partial X) \rightarrow$ End $\mathbf{E}$ which is an algebra homomorphism on each fiber and which covers the identity:

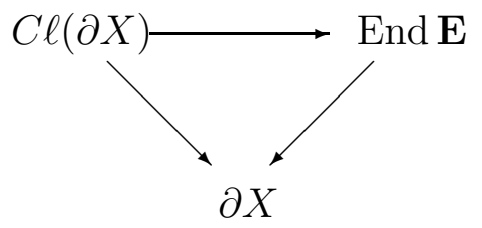

is commutative. Denote by $\mathcal{S}$ the spin bundles associated to the two half-spin representation of $\operatorname{Spin}(\operatorname{dim} \partial X)$; then $\mathcal{S}=\mathcal{S}^{+} \oplus \mathcal{S}^{-}$where $\mathcal{S}^{ \pm}$are half-spin bundles. $\nabla$ induces a connection on $\mathcal{S} \otimes \mathbf{E}$ which is compatible with both the metric and the $\mathbf{Z}_{2}-$ grading. The latter connection canonically defines a Dirac operator $\mathcal{D}_{\partial X}: C^{\infty}\left(\mathcal{S}^{+} \otimes \mathbf{E}\right) \rightarrow C^{\infty}\left(\mathcal{S}^{-} \otimes \mathbf{E}\right)$ described by 


$$
\mathcal{D}_{\partial X}: C^{\infty}\left(\mathcal{S}_{\mathbf{E}}^{+}\right) \stackrel{\nabla}{\longrightarrow} C^{\infty}\left(T^{*} \partial X \otimes \mathcal{S}_{\mathbf{E}}^{+}\right) \stackrel{\mathcal{H}}{\longrightarrow} C^{\infty}\left(\mathcal{S}_{\mathbf{E}}^{-}\right),
$$

where $\mathcal{H}: T^{*} \partial X \longrightarrow \operatorname{Hom}\left(\mathcal{S}_{\mathbf{E}}^{+}, \mathcal{S}_{\mathbf{E}}^{-}\right)$denotes the Clifford multiplication and $\mathcal{S}_{\mathbf{E}}^{ \pm} \stackrel{\text { def }}{=} \mathcal{S}^{ \pm} \otimes$ E. Then $\operatorname{dim} \operatorname{Ker}^{+} \mathcal{D}_{\partial X}=\operatorname{dim} \operatorname{Ker}^{-} \mathcal{D}_{\partial X}$ and the boundary condition is an isometry $I: \operatorname{Ker}^{+} \mathcal{D}_{\partial X} \rightarrow \operatorname{Ker}^{-} \mathcal{D}_{\partial X}$. Therefore the invariant (2.1) is defined and we have $\tau_{X} \in$ $\operatorname{Det}_{\partial X}^{-1}$, where $\operatorname{Det}_{\partial X}$ is the determinant line of the Dirac operator $\mathcal{D}_{\partial X}$ on the boundary: $\operatorname{Det}_{\partial X}=\left(\operatorname{Det~Ker}^{-} \mathcal{D}_{\partial X}\right) \otimes\left(\operatorname{Det}_{K^{+}{ }^{+}} \mathcal{D}_{\partial X}\right)^{-1}$.

Let a family of Riemannian manifolds is a smooth fiber bundle $\pi: X \rightarrow Z$ together with a metric on the relative tangent bundle $T(X / Z)$ which endowed with spin structure. Also we asume that the Riemannian metrics on the fibers are products near boundary. The determinant lines carries the Quillen metric and a canonical connection $\nabla$ [17] and the exponentiated eta invariant is a smooth section $\tau_{X / Z}: Z \rightarrow \operatorname{Det}_{\partial X / Z}^{-1}$.

We mention here two basic results on this invariant, namely variation and curvature formulas.

Theorem 2.1. (Dai and Freed [19], Theorem 1.9.) The covariant derivative of the exponentiated eta invariant is

$$
\nabla \tau_{X / Z}=2 \pi \sqrt{-1}\left[\int_{X / Z} \widehat{A}\left(\Omega^{X / Z}\right)\right]_{(1)} \cdot \tau_{X / Z},
$$

where $\Omega^{X / Z}$ is the Riemannian curvature of $X \rightarrow Z, \widehat{A}$ is the usual polynomial

$$
\widehat{A}(\Omega)=\sqrt{\operatorname{det}\left(\frac{\Omega / 4 \pi}{\sinh \Omega / 4 \pi}\right)},
$$

$\operatorname{Tr} e^{\sqrt{-1} \Omega / 2 \pi}=\operatorname{ch}(\Omega)$ and simbol (1) denotes the 1-form piece of the differential form.

Note that for a family of closed manifolds this is a result of Atiyah-Patodi-Singer. The fiber of $X \rightarrow Z$ we denote as $X / Z$ and let it will be a closed manifold. Let $K O(X)$ is the group of virtual real vector bundles on $X$ up to equivalence and $K O^{-N}(X) \subset K O\left(\mathbf{S}^{N} \otimes X\right)$, $N=\operatorname{dim}(X / Z)$, is the subspace of isomorphism classes of virtual bundles trivial on $\mathbf{S}^{N} \vee X$. The spin structure on the fibers can be determined by a pushforward map $\pi_{!}^{X / Z}: K O(X) \rightarrow K O^{-N}(Z)$. Let $E \rightarrow X$ is a real vector bundle; the family of Dirac operators on $X / Z$ coupled to $E$ has an index in $K O^{-N}(Z)$, which equals [24] $\pi_{!}^{X / Z}([E])$, where $[E] \in K O(X)$ is the isomorphism class of $E$.

In general for Dirac operators coupled to complex bundles in K-theory one can express the Chern character of the index in terms of Chern character of $E$ by means of the formula [25:

$$
\operatorname{ch} \pi_{!}^{X / Z}([E])=\pi_{*}^{X / Z}(\hat{A}(X / Z) \operatorname{ch}(E)),
$$

where $\pi_{*}$ is the pushforward map in rational cohomology. 
Let the fibers $X / Z$ are closed. Then the determinant line bundle $\operatorname{Det} \mathcal{D}^{X / Z}(E)$ is a well-defined as a smooth line bundle (it carries a canonical metric and connection) [26]. The complex Dirac operator for the odd dimensional fibers $X / Z$ is self-adjoint and there is a geometrical invariant $\xi_{X / Z}(E): Z \rightarrow \mathbf{R} / \mathbf{Z}$ defined by Atiyah-Patodi-Singer,

$$
2 \xi_{X / Z}=(\eta(0)+\operatorname{dimKer} \mathcal{D})_{X / Z}
$$

We have

$$
\tau_{X / Z}(E)=\exp \left(2 \pi \sqrt{-1} \xi_{X / Z}\right): Z \rightarrow \mathbf{T},
$$

where $\mathbf{T} \subset \mathbf{C}$.

Theorem 2.2. (Bismut and Freed [27], Theorem 1.21.) The 2-form curvature of the determinant line bundle is

$$
\Omega^{\operatorname{Det} \mathcal{D}^{X / Z}(E)}=\left[2 \pi \sqrt{-1} \int_{X / Z} \hat{A}\left(\Omega^{X / Z}\right) \operatorname{ch}\left(\Omega^{E}\right)\right]_{(2)} \in \Omega^{2}(Z),
$$

where $\Omega^{X / Z}$ and $\Omega^{E}$ are the curvature forms.

Let $X \rightarrow \mathbf{S}^{1}$ is a loop of manifolds in this geometric setup. A metric and spin structure on $X$ could be induced by a metric and boundary spin structure on $\mathbf{S}^{1}$. The holonomy of the determinant line bundles around loop takes the form

$$
\operatorname{hol} \operatorname{Det} \mathcal{D}^{X / \mathbf{S}^{1}}(E)=\mathrm{a}-\lim \tau_{X}^{-1}(E),
$$

where a-lim is the adiabatic limit, i.e. the limit as the metric on $\mathbf{S}^{1}$ blows up $(\varepsilon \rightarrow 0$ : $\left.\mathrm{g}_{\mathbf{S}}^{1} \rightarrow \mathrm{g}_{\mathbf{S}}^{1} \varepsilon^{-2}\right)$. For the flat determinant line bundles no adiabatic limit is required. As we pointed out before, Eq. (2.10) is the global anomaly formula [21, 26].

\section{Index of the Dirac operator}

Calculating index of the Dirac operator we shall follow the lines of paper [28] and consider special case $G=S O_{1}(2 n, 1), K=S O(2 n)$. The complexified Lie algebra $\mathrm{g}=\mathrm{g}_{0}^{\mathbf{C}}=$ $s o(2 n+1, \mathbf{C})$ of $G$ is of Cartan type $B_{n}$ with Dynkin diagram

$$
\underbrace{\bigcirc-\bigcirc-\bigcirc \cdots \bigcirc-\bigcirc}_{2 n \text { nodes }}=\bigcirc \text {. }
$$

The standard systems of positive roots $\triangle^{+}, \triangle_{k}^{+}$for $\mathrm{g}$ and $k=k_{0}^{\mathbf{C}}$ - the complexified Lie algebra of $K$, with respect to a Cartan subgroup $H$ of $G, H \subset K$, are given by 


$$
\triangle^{+}=\left\{\varepsilon_{i} \mid 1 \leq i \leq n\right\} \bigcup \triangle_{k}^{+}
$$

where

$$
\triangle_{k}^{+}=\left\{\varepsilon_{i} \pm \varepsilon_{j} \mid 1 \leq i<j \leq n\right\}
$$

and

$$
\triangle_{n}^{+} \stackrel{\text { def }}{=}\left\{\varepsilon_{i} \mid 1 \leq i \leq n\right\}
$$

is the set of positive non-compact roots. Here

$$
\left(\varepsilon_{i}, \varepsilon_{j}\right)=\frac{\delta_{i j}}{2(2 n-1)},
$$

(, ) being the Killing form of $\mathrm{G}$. Let $h_{0}$ be the Lie algebra of $H$ and let $h_{\mathbf{R}}^{*}=$ $\operatorname{Hom}\left(\sqrt{-1} h_{0}, \mathbf{R}\right)$ be the dual space of the real vector space $\sqrt{-1} h_{0}$. Thus the $\left\{\varepsilon_{i}\right\}_{i=1}$ are an $\mathbf{R}$ - basis of $h_{\mathbf{R}}^{*}$. Of interest are the integral elements $f$ of $h_{\mathbf{R}}^{*}$ :

$$
f \stackrel{\text { def }}{=}\left\{\mu \in h_{\mathbf{R}}^{*} \mid \frac{2(\mu, \alpha)}{(\alpha, \alpha)} \in \mathbf{Z}, \forall \alpha \in \Delta^{+}\right\}
$$

Using Eq. (3.5) we have

$$
\begin{gathered}
\frac{2\left(\mu, \varepsilon_{i}\right)}{\left(\varepsilon_{i}, \varepsilon_{i}\right)}=2 \mu_{i} \quad \text { for } 1 \leq i \leq n, \\
\frac{2\left(\mu, \varepsilon_{i} \pm \varepsilon_{j}\right)}{\left(\varepsilon_{i} \pm \varepsilon_{j}, \varepsilon_{i} \pm \varepsilon_{j}\right)}=\mu_{i} \pm \mu_{j} \quad \text { for } \quad 1 \leq i<j \leq n,
\end{gathered}
$$

where we shall write $\mu=\sum_{j=1}^{n} \mu_{j} \varepsilon_{j}$ for $\mu \in h_{\mathbf{R}}^{*}, \mu_{j} \in \mathbf{R}$. Then clearly

$$
\begin{array}{ll}
f=\left\{\mu \in h_{R}^{*} \mid\right. & 2 \mu_{i} \in \mathbf{Z} \quad \text { for } 1 \leq i \leq n \\
& \left.\mu_{i} \pm \mu_{j} \in \mathbf{Z} \quad \text { for } 1 \leq i<j \leq n\right\} .
\end{array}
$$

Let $\delta_{k}=\frac{1}{2} \sum_{\alpha \in \triangle_{k}^{+}} \alpha, \delta_{n}=\frac{1}{2} \sum_{\alpha \in \triangle_{n}^{+}} \alpha, \delta=\delta_{k}+\delta_{n}=\frac{1}{2} \sum_{\alpha \in \triangle^{+}} \alpha$. Then $\delta_{k}=\sum_{i=1}^{n}(n-$ i) $\varepsilon_{i}, \delta_{n}=\frac{1}{2} \sum_{i=1}^{n} \varepsilon_{i}, \delta=\sum_{i=1}^{n}\left(n-i-\frac{1}{2}\right) \varepsilon_{i}$ are all integral. Elements $\mu$ of $f$ corresponds to characters $e^{\mu}$ of $H$. Following the paper 28] we fix once and for all $\mu \in f$ which is $\triangle_{k}^{+}-$ dominant: $(\mu, \alpha) \geq 0$ for $\alpha \in \triangle_{k}^{+}$. For us, in concrete terms, this means the following: let $\mu=\left(\mu_{1}, \ldots, \mu_{n}\right)$ be a sequence of real numbers such that 
(i) $2 \mu_{i} \in \mathbf{Z}$ for $1 \leq i \leq n$ and $\mu_{i} \pm \mu_{j} \in \mathbf{Z}$ for $1 \leq i<j<n$ (i.e. $\mu$ is integral).

(ii) $\left|\mu_{n}\right| \leq \mu_{n-1} \leq \mu_{n-2} \leq \ldots \leq \mu_{2} \leq \mu_{1}$ and either every $\mu_{i} \in \mathbf{Z}$ or every $\mu_{i}$ is half an odd integer.

Note that in fact (ii) $\rightarrow$ (i) so that we may drop condition (i) (only for $G=S O_{1}(2 n, 1)$ since in general (ii) does not imply (i)). Then $\mu+\delta_{n}$ does define a character of $H$ as has been required in [28] and by their construction ( $\mu$ be the highest weight of an irreducible $k$ - module $V_{\mu}$ ) there is a twisted Dirac operator $\mathcal{D}_{\Gamma \backslash X}^{\chi}$ on a vector bundle over $\Gamma \backslash G / K$ for $\Gamma$ a discrete subgroup of $G$ for $\Gamma$ satisfying the conditions of their paper.

Thus $\Gamma \backslash G$ need not be compact, but one requires of course that $\operatorname{Vol}(\Gamma \backslash G)<\infty$. The paper [28] also requires that a choice $\psi$ of positive root system for $\left(\mathrm{g}, h=h_{0}^{\mathrm{C}}\right)$ be chosen such that $\triangle_{k}^{+} \subset \psi$ and such that $\mu+\delta_{k}$ is $\psi$ - dominant, i.e. $\left(\mu+\delta_{k}, \alpha\right) \geq 0 \forall \alpha \in \psi$ (condition (1.3.1) of [28] where $\rho_{c}=\delta_{k}, \Phi=\triangle \stackrel{\text { def }}{=} \triangle^{+} \cup\left(-\triangle^{+}\right), \Phi_{k}=\triangle_{k}^{+}, \Phi_{n}=$ $\left.\triangle_{n}^{+}, T=H\right)$. Here we choose $\psi=\triangle^{+}$provided $\mu_{n} \geq 0$, which we now assume; see (ii).

\subsection{Twisted Dirac operator}

In summary we assume the following. $\mu=\left(\mu_{1}, \ldots, \mu_{n}\right)$ is a sequence of real numbers, and

(iii) $0 \leq \mu_{n} \leq \mu_{n-1} \leq \mu_{n-2} \leq \cdot \leq \mu_{2} \leq \mu_{1}$, where either every $\mu_{j} \in \mathbf{Z}$ or every $\mu_{j}$ has the form $\mu_{j}=n_{j}+\frac{1}{2}$ for some $n_{j} \in \mathbf{Z}$.

Then we have a twisted Dirac operator $\mathcal{D}_{\Gamma \backslash X}^{\chi}$ over $\Gamma \backslash G / K$.

Theorem 3.1 (Barbasch and Moscovici 28] for the case $G=S O_{1}(2 n, 1), n \geq 2$ ). For a suitable normalization of Haar measure on $G$, and for $\mu$ satisfying condition (iii) one has

$$
\operatorname{Index} \mathcal{D}_{\mu, \Gamma}^{+}=\operatorname{Vol}(\Gamma \backslash G) \frac{\prod_{\alpha \in \Delta^{+}}\left(\mu+\delta_{k}, \alpha\right)}{\prod_{\alpha \in \Delta_{k}^{+}}\left(\delta_{k}, \alpha\right)} .
$$

To make this explicit we must express $P_{k} \stackrel{\text { def }}{=} \prod_{\alpha \in \Delta_{k}^{+}}\left(\delta_{k}, \alpha\right)$ and $P \stackrel{\text { def }}{=} \prod_{\alpha \in \Delta+}(\mu+\delta, \alpha)$ directly in terms of the real numbers $\mu_{1}, \ldots, \mu_{n}$.

Tedious calculation gives the final result for $\mu=\left(\mu_{1}, \ldots, \mu_{n}\right) \in \mathbf{R}^{n}$ subject to condition (iii) (for suitable Haar measure on $G$ ). Namely, the $L^{2}$ - index of twisted Dirac operator $\mathcal{D}_{\mu, \Gamma}^{+}$is equal to

$$
\begin{gathered}
\operatorname{Index} \mathcal{D}_{\mu, \Gamma}^{+}=\operatorname{Vol}(\Gamma \backslash G) \frac{P}{P_{k}}=\frac{\operatorname{Vol}(\Gamma \backslash G)}{\left[2(2 n-1)^{n}\right]} \\
\times \frac{\prod_{i=1}^{n}\left(\mu_{i}+n-i\right) \prod_{1 \leq i<j \leq n}\left(\mu_{i}+\mu_{j}+2 n-i-j\right)\left(\mu_{i}-\mu_{j}-i+j\right)}{\prod_{1 \leq i<j \leq n}(2 n-i-j)(-i+j)} .
\end{gathered}
$$




\section{Concluding remarks}

There are many applications of these formulae. We note some of them. For example, the results obtained in the paper could be used for calculation of Chern-Simons functional related to a real hyperbolic 3-manifold [29, 30, 31, 32, 33, 34]:

$$
\begin{aligned}
C S\left(A_{\chi}\right) & =\frac{1}{2 \pi \sqrt{-1}} \log \left[\frac{Z(0, \mathcal{D})^{\operatorname{dim} \chi}}{Z\left(0, \mathcal{D}_{\chi}\right)}\right]+\left(\operatorname{dim} \chi \operatorname{Index} \mathcal{D}_{A_{\chi_{0}}}-\operatorname{Index} \mathcal{D}_{A_{\chi}}\right) \\
& +\frac{1}{2}\left(\operatorname{dim} \chi \operatorname{dimKer} \mathcal{D}-\operatorname{dimKer} \mathcal{D}_{\chi}\right)_{\Gamma \backslash \mathbf{H}^{3}} .
\end{aligned}
$$

Here $Z\left(s, \mathcal{D}_{\chi}\right)$ is a Selberg type (Shintani) zeta function [35, 36] and any 1-dimensional representation $\chi$ of $\Gamma$ factors through a representation $H^{1}(X, \mathbf{Z})$, and $\chi_{0}$ is a trivial representation.

Eq. (2.10) is known as the global anomaly formula [21, 26]. Global anomalies in the worldsheet path integral of string theory in the presence of $D$ - branes has ben studied in [25]. Recently agreement between partition functions of IIA string theory and $M$ - theory (a derivation $K$ - theory from $M$ - theory) was found in [37]. One can calculate also the phase of the one-loop contributions from instantons to the membrane superpotential [38].

In all of these edxamples the lolonomy of the determinant line bundles (and the index of a Dirac operator, for example (3.11)) play very important role.

\section{Acknowledgements}

A.A.B. very grateful to S.D. Odintsov and F.L. Williams for helpful discussion. The work of A.A.B. was supported in part by the Russian Foundation for Basic Research (grant No. 01-02-17157). A.E.G. thanks CNPq for partial support.

\section{References}

[1] R. Dijkgraaf and E. Witten, Commun. Math. Phys. 129 (1990) 393.

[2] R. Kirby And P. Melvin, Invent. Math. 105 (1991) 473.

[3] D.S. Freed And R.E. Gompf, Commun. Math. Phys. 141 (1991) 79.

[4] L.C. Jeffrey, Commun. Math. Phys. 147 (1992) 563.

[5] S.K. Rama and S. Sen, Mod. Phys. Lett. A 8 (1993) 2285.

[6] L. Rozansky, Commun. Math. Phys. 171 (1995) 279. 
[7] L. Rozansky, Commun. Math. Phys. 175 (1996) 275.

[8] M. Blau and G. Thompson, Commun. Math. Phys. 152 (1993) 41.

[9] N. Seiberg and E. Witten, Nucl. Phys. B 426 (1994) 19.

[10] E. Witten, Math. Res. Lett. 1 (1994) 769.

[11] S. Nojiri And S.D. Odintsov, Phys. Lett. B 471 (1999) 155.

[12] L. Rozansky and E. Witten, Selecta Math. 3 (1997) 401.

[13] S. Axelrod And I.M. Singer, J. Diff. Geom. 39 (1994) 173.

[14] E. Elizalde, S.D. Odintsov, A. Romeo, A.A. Bytsenko and S. Zerbini, "Zeta Regularization Techniques with Applications", World Scientific (1994).

[15] A. A. Bytsenko, G. Cognola, L. Vanzo and S. Zerbini, Phys. Rep. 266 (1996) 1.

[16] D. Quillen, Funk. Anal. i Prilozen. 19 (1985) 37.

[17] J.M. Bismut And D.S. Freed, Commun. Math. Phys. 106 (1986) 159.

[18] J.M. Bismut And D.S. Freed, Commun. Math. Phys. 107 (1986) 103.

[19] X. Dai And D.S. Freed, J. Math. Phys. 35 (1994) 5155.

[20] D.S. Freed, "Determinant Lines Bundles Revisted", dg-ga/9505002.

[21] E. Witten, Commun. Math. Phys. 100 (1985) 197.

[22] M.F. Atiyah, V.K. Patodi and I.M. Singer, Math. Proc. Cambridge Philos. Soc. 77 (1975) 43.

[23] H.B. Lawson and M.L. Michelson, "Spin Geometry", Princeton Mathematical Series 38, Princeton Univ. Press, Princeton (1989).

[24] M.F. Atiyah, V.K. Patodi and I.M. Singer, Math Proc. Cambridge Philos. Soc. v. 79 (1976) 71.

[25] D.S. Freed And E. Witten, "Anomalies in String Theory with D-Branes", hepth/9907189.

[26] J.M. Bismut And D.S. Freed, Commun. Math. Phys. v. 107 (1986) 159.

[27] J-M. Bismut and D.S. Freed, Comm. Math. Phys. 107 (1986) 103.

[28] D. Barbasch and H. Moscovici, J. Fuct. Anal. 53 (1983) 151.

[29] A.A. Bytsenko, L. Vanzo and S. Zerbini, Nucl. Phys. B 505 (1997) 641. 
[30] A.A. Bytsenko, A.E. Gonçalves and W. Da Cruz, Mod. Phys. Lett. A 13 (1998) 2453.

[31] A.A. Bytsenko, L. Vanzo and S. Zerbini, Phys. Lett. B 459 (1999) 535.

[32] A.A. Bytsenko, M.C. Falleiros and A.E. Gonçalves, JETP Lett. 70 (1999) 67.

[33] A.A. Bytsenko, A.E. Gonçalves and F.L. Williams, "Chern-Simons Invariants of Closed Hyperbolic 3-Manifolds", Proceedings of the 1999 Londrina Winter School "Mathematical Methods in Physics", Eds. A.A. Bytsenko and F.L. Williams, World Sci., (1999) 70.

[34] A.A. Bytsenko, A.E. Gonçalves and B.M. Pimentel, "Invariants of ChernSimons Theory Associated with Hyperbolic Manifolds", Proceedings of the 2000 Londrina Workshop "Geometrical Aspects of Quantum Fields", Eds. A.A. Bytsenko, A.E. Gonçalves and B.M. Pimentel, World Sci., (2000) 31.

[35] J.J. Millson, Ann. Math. 108 (1978) 1.

[36] H. Moscovici and R.J. Stanton, Invent. Math. 95 (1989) 629.

[37] D.-E. Diaconescu, G. Moore and E. Witten, "E $E_{8}$ Gauge Theory, and a Derivation of K-Theory from M-Theory", hep-th/0005090.

[38] J.A. Harvey and G. Moore, "Superpotentials and Membrane Instantons", hepth/9907026. 